\title{
Minimum Length Scheduling for Multi-Cell Wireless Powered Communication Networks
}

\author{
Elif Dilek Salik, Aysun Gurur Onalan, and Sinem Coleri \\ Department of Electrical and Electronics Engineering \\ Koc University, Istanbul, Turkey \\ Email: \{esalik, aonalan17, scoleri\}@ku.edu.tr
}

\begin{abstract}
We consider a wireless powered, harvestthen-transmit communication network, which consists of multiple, single antenna, energy and information access points (APs) and multiple, single antenna users with energy harvesting capabilities and rechargeable batteries, and allows simultaneous information transmission. We formulate the joint power control and scheduling problem with the objective of minimizing the total schedule length, subject to the constraints on the minimum amount of data to be sent by the users to the APs, and the maximum transmit power for the information transmission. This problem is a nonlinear and non-convex, mixed integer programming problem for which there is no known polynomial time algorithm. The proposed heuristic algorithm is based on, first, finding the solution for a fixed energy harvesting time and then searching for the optimal energy harvesting time that minimizes the total schedule length. For the former, a scheduling problem is formulated as an integer programming problem, which we solve with Branch and Price based methods upon solving the power control problem separately. Simulation results demonstrate that the proposed algorithm outperforms previously proposed time minimization algorithms that do not consider simultaneous transmission scenarios up to $3.5 \%$ for larger AP power, $25.4 \%$ for tighter maximum transmit power limit, and $6.5 \%$ for greater number of users per AP.
\end{abstract}

\section{INTRODUCTION}

The issue of renewing or replacing batteries in traditional sensor networks is potentially solved with energy harvesting $(\mathrm{EH})$. Wireless powered communication networks (WPCNs) have gained great interest lately, since EH users in WPCNs are provided with controllable amounts of energy via radio frequency (RF) signals broadcast by an access point (AP) [1].

WPCNs have been studied with the objective of transmission time minimization to ensure the delay performance of time critical applications while satisfying the users' data demands. Harvest-then-transmit (HTT) is an often applied protocol in WPCNs, in which, users harvest energy at the beginning of a transmission frame in the downlink (DL), and then, the rest of the frame is allocated for information transmission (IT) in the uplink (UL). Packet scheduling is examined to minimize the

This work is supported by Scientific and Technological Research Council of Turkey Grant \#117E241. delay of a pair of users transmitting packets to each other while switching between EH and IT [2]. Total time is minimized for full-duplex WPCNs for continuous [3] and discrete [4] rate assumptions. A half-duplex WPCN is studied for total time minimization with the minimum amount of information to be transmitted in [5], and, additionally with the maximum UL power limit in [6].

All of the aforementioned studies assume single AP WPCNs. However, the number of users and connected areas have been increasing in practice. A single AP is not enough to cover and serve the expected high amount of connected devices. Deploying multiple APs and having a multi-cell like structure is, therefore, inevitable. Optimal transmit power allocation subject to a power budget for a multiple user and multiple RF energy transmitter network working with orthogonal frequency division multiplexing protocol is studied for the sum and common throughput maximization [7]. Sum-throughput is maximized by jointly optimizing intra-cell time allocation and inter-cell load balancing for a multi-cell WPCN which adopts a fixed inter-cell interference at APs [8]. Weighted sum-rate is maximized by asynchronous time allocation for EH and IT in a multi-cell network where each cell only has one user [9]. [10] optimizes time allocation to maximize the minimum and sum throughput in a multi-cell HTT WPCN with load coupling and long term average interference, while [11] also integrates self interference cancellation at APs. All these works in multi-cell WPCNs aim throughput maximization. To the best of our knowledge, there is no work in literature that considers delay critical multi-cell WPCNs and minimizes total schedule length.

In this paper, we consider a HTT WPCN with multiple half-duplex APs and multiple users where users harvest energy from the signal broadcast by all APs for their IT. The main contributions of this paper are as follows:

- A novel optimization problem is formulated for the joint optimization of EH time, IT times and UL power allocations, and scheduling of users to minimize the total schedule length of the network. The problem is constrained by the assignment of the users to the time slots and their data amounts 
to send in the UL with the UL transmit power limit. This is a mixed-integer, non-linear, non-convex problem with no known polynomial time algorithm.

- We think of this problem as a two-tier problem, such that, first, EH time is fixed and the problem is solved for optimal IT times and schedule, and then, as the remaining problem of searching the optimal EH time that minimizes the total schedule length is non-convex, we apply a heuristic search algorithm, simulated annealing.

- The first tier is further divided into two problems as power control and scheduling, which are formulated and solved. Fast Iterative Power Control Algorithm for EH Networks, FIPCA-EH, to solve the nonconvex power control problem and Branch and Price Based Heuristic Algorithm for EH Networks, $\mathrm{BP}-\mathrm{EH}$, to solve the integer programming (IP) scheduling problem are proposed. The proposed algorithm which includes FIPCA-EH, BP-EH and simulated annealing algorithms is named as MultiAP Multi-User Scheduling Algorithm, MAMUSA.

- We demonstrate via simulations that MAMUSA outperforms the previously proposed algorithms for asynchronous transmission for larger AP power in DL EH, tighter maximum power limit in UL IT, and greater number of users as all these cases favor simultaneous transmission.

The paper is organized as follows: Section II describes the system model and the assumptions used throughout the paper. Section III formulates the minimum length scheduling problem and gives the solution strategy. Sections IV and V formulates power control and scheduling problems, respectively, and discuss their solution. The performance of the proposed algorithm is analysed in Section VI. Section VII concludes the paper.

\section{System Model And Assumptions}

- We consider a multi-cell WPCN applying HTT protocol with $N$ users and $C$ APs. $L^{c}$ denotes the set of users that are under the coverage of the $c$-th AP. All users and APs are equipped with single antenna and operating over the same frequency band.

- The users are assumed to only have rechargeable batteries with low energy storage and high self discharge rate, e.g., super-capacitors [12]. Thus, the harvested energy in a frame is used for IT within that frame, not stored for later use. A transmission frame is partitioned into variable length time slots, $\tau^{m}$ s. During the first time slot of a frame, $\tau^{0}$, APs broadcast DL signal to the users for EH. The remaining time slots are assigned for the IT in the UL from the users to their corresponding APs.

- At each time slot allocated for UL IT, only one user in $L^{c}$ transmits to the $c$-th $\mathrm{AP}, \forall c$. However, the users that are connected to the different APs can transmit information in the UL simultaneously. A transmission frame consists of, at most, $N+1$ time slots, one for $\mathrm{EH}$ and $N$ for IT, if no simultaneous transmission occurs, i.e., users transmit one-by-one.

- We assume to know the connected AP-user pairs, referred as links, when UL transmission occurs. Each AP is responsible of maintaining the synchronization of the users that it covers and inform them about their current schedule.

- $U_{i}$ has to send a certain amount of data in a frame, denoted as $D_{i}$, which is constant and positive.

- We use continuous rate transmission model, in which Shannon's channel capacity formulation for an Additive White Gaussian Noise (AWGN) wireless channel is used in the calculation of the transmission rate at the $m$-th time slot as a function of SINR as

$$
W \times \log \left(1+\frac{1}{W} \times \frac{P_{i}^{m} g_{i i}}{N_{0}+\sum_{j \neq i} P_{j}^{m} g_{j i}}\right)
$$

where $P_{i}^{m}$ is the transmission power of the $i$-th user at the $m$-th time slot, $g_{i i}$ and $g_{j i}$ denote the channel gain of link $i$ and the channel gain from $U_{j}$ to the AP in the link $i$, i.e., the AP that the $U_{i}$ is connected to. The channels are block-fading and all the channel gains are assumed to be perfectly known by the APs.

- APs transmit with maximum power denoted by $P_{A}$. Power transmitted to the $U_{i}$ by all APs is denoted by $P_{i}^{T}$. The harvested energy at $U_{i}$ at a unit time becomes $E_{i}=\zeta_{i} P_{i}^{T}$, where $\zeta_{i} \in(0,1)$ is the constant EH efficiency of $U_{i}$.

- The power radiated by $U_{i}$ is limited by an upper limit, mostly due to the hardware limitations of the power amplifier, denoted as $P_{i}^{\max }$, and further limited by the amount of the harvested energy, $E_{i}$.

\section{Optimization PRoblem}

The joint optimization of transmit power, time allocation and the scheduling of users with the aim of minimizing the total EH and IT time of a HTT WPCN with multiple users and multiple APs subject to the constraints that each user has certain amount of data to send to its corresponding AP and a maximum allowable transmit power is mathematically formulated. 


\section{A. Problem Formulation}

$$
\begin{aligned}
& \min \sum_{m=0}^{N} \tau^{m} \\
& \text { s.t. } \quad \sum_{m=1}^{N} b_{i}^{m}=1, \forall i=1, \ldots, N \text {, } \\
& \sum_{i \in L^{c}} b_{i}^{m} \leq 1, \forall m=1, \ldots, N, \forall c=1, \ldots, C, \\
& P_{i}^{m} \leq P_{i}^{\max } b_{i}^{m}, \forall i=1, \ldots, N, \forall m=1, \ldots, N, \\
& P_{i}^{m} \tau^{m} \leq E_{i} \tau^{0}, \forall i=1, \ldots, N, \forall m=1, \ldots, N, \\
& \sum_{m=1}^{N} W \log _{2}\left(1+\frac{P_{i}^{m} g_{i i}}{W\left(N_{0}+\sum_{j=1, j \neq i}^{N} P_{j}^{m} g_{j i}\right)}\right) \tau^{m} \\
& \geq D_{i}, \quad \forall i=1, \ldots, N, \quad(2 \mathrm{f}) \\
& \text { vars } \quad \tau^{m} \geq 0, \forall m=0, \ldots, N \text {, } \\
& b_{i}^{m} \in\{0,1\}, \forall i=1, \ldots, N, \forall m=1, \ldots, N, \\
& P_{i}^{m} \geq 0, \forall i=1, \ldots, N, \forall m=1, \ldots, N \text {. }
\end{aligned}
$$

The objective of Problem (2) is to minimize the total schedule length for both the EH and ITs, where $\tau^{m}$ is the length of the $m$-th time slot, $\forall m \in[0, N] . b_{i}^{m}$ is a binary variable which takes value 1 if the $i$-th user is assigned to the $m$-th time slot, and 0 otherwise. The decision variables of the problem are the time slot length, $\tau^{m}$, assignment of the users to the time slots, $b_{i}^{m}$, and UL transmit powers of the users, $P_{i}^{m}$. Eq. (2b) ensures that each user is assigned to one and only one time slot. Eq. (2c) guarantees that at most one user is assigned to an AP at a given time slot. Eq. (2d) defines an upper bound on the maximum allowable transmit power of the $U_{i}$ if it is assigned to the $m$-th time slot. If $U_{i}$ is not assigned at the $m$-th time slot, then, $P_{i}^{m}$ is forced to be 0 . Eq. (2e) ensures that at least the amount of energy consumed by $U_{i}$ at the $m$-th time slot for IT is harvested by $U_{i}$ during the $\mathrm{EH}$ time, $\tau^{0}$. The data requirement that $U_{i}$ has to meet during the time slot it is assigned to is given in Eq. (2f). Eqs. (2g) - (2i) are the non-negativity and integrality constraints of the decision variables.

Due to the non-convexity of Eq. (2e) and the nonlinearity of Eq. (2f), Problem (2) is a mixed integer, nonlinear, non-convex problem for which there is no known polynomial time algorithm [13].

\section{B. Solution Strategy}

For traditional wireless communication networks without EH users, Problem (2) reduces to a minimum length scheduling problem, which is studied in [14]. Therefore, we also propose a solution strategy based on the decomposition of the power control and scheduling problems as in [14] while adapting the related algorithms to the additional EH constraints that Problem (2) has. Further, we perform a non-convex search on the optimal $\mathrm{EH}$ time as total schedule length is a non-convex function of $\mathrm{EH}$ time due to the non-convexity originated from the EH constraints.
Notice that, for a fixed $\tau^{0}$, the UL transmit power and time slot length variables of Problem (2) account only for one time slot at a time. Hence, to achieve the minimum schedule length, the length of each time slot should be minimized independent of one another.

We first formulate a power control problem which minimizes the time slot length required for the concurrent transmission of a set of simultaneously active links in Section IV. And, we propose FIPCA-EH algorithm to solve the power control problem. Then, an integer programming (IP) scheduling problem is formulated in Section V, which minimizes the total schedule length given the minimum time slot lengths corresponding to every possible user allocation subset which is calculated by FIPCA-EH. A Branch and Price (BP) and Column Generation Method (CGM) based heuristic algorithm is proposed to solve the large scale IP scheduling problem, named as BP-EH.

Finally, given the minimized total IT times corresponding to fixed EH times calculated by the power control and scheduling problems, we propose a search algorithm to find the optimal EH time, which minimizes the total schedule length. Since the total schedule length with respect to EH time exposes non-convex behavior, a simulated annealing based search algorithm is performed. Simulated annealing helps finding a global optimal solution in the presence of a large number of local optima by jumping around local optima. The algorithm selects the improving directions with a certain probability while decreasing the extent of its search to converge to an optimal solution [15].

The overall solution procedure is named as Multi-AP Multi-User Scheduling Algorithm (MAMUSA).

\section{Power Control Problem}

The power control problem minimizes the length of a time slot given the data requirements of the active users in that slot. Let $S$ be the set of active links in a time slot. The superscripts of the variables indicating their time slots are omitted. Hence, $\tau$ denotes the length of the time slot to be minimized.

$$
\begin{aligned}
& \min \tau \\
& \text { s.t. } P_{i} \leq P_{i}^{\max }, \forall i \in S \text {, } \\
& P_{i} \tau \leq E_{i} \tau^{0}, \forall i \in S, \\
& W \log _{2}\left(1+\frac{P_{i} g_{i i}}{W\left(N_{0}+\sum_{j=1, j \neq i}^{N} P_{j} g_{j i}\right)}\right) \tau \geq D_{i}, \\
& \forall i \in S, \quad(3 \mathrm{~d}) \\
& \text { vars } \tau \geq 0 \text {, } \\
& P_{i} \geq 0, \quad \forall i \in S \text {. }
\end{aligned}
$$

The power control problem given in Eq. (3) is a nonconvex and nonlinear optimization problem due to the non-convexity of Eq. (3c) and nonlinearity of Eq. (3d).

\section{A. Solution}

For the solution of the power control problem given in Eq. (3), we propose Fast-Iterative Power Control 
Algorithm for EH Networks (FIPCA-EH). FIPCA-EH performs an intelligent bi-section search for the minimum feasible value of $\tau$, where the feasibility of the midpoint of the search interval is evaluated at each iteration.

For a fixed $\tau$, Problem (3) reduces to determining whether there exists a set of transmit power assignments within the limits of the harvested energy amount by the links in the set $S$, such that the required amounts of data can be transmitted. Note that the optimal transmission time for all the links assigned to the same time slot is equal, which is proven by Theorem 2 in [14]. The existence of the transmit power allocation for the required data transmission is checked by PerronFrobenius conditions [14] as follows: Let $B_{S}$ be $|S| \times|S|$ relative channel gain matrix, such that the value of the $i^{\text {th }}$ row and $j^{\text {th }}$ column of $B_{S}$ is $g_{j i} / g_{i i}$ for $i \neq j$ and 0 for $i=j$. Let $D_{S}$ be $|S| \times|S|$ diagonal matrix, such that $i^{t h}$ diagonal entry of $D_{S}$ is $\beta_{i}$, where $\beta_{i}=W\left(2^{D_{i} / W \tau}-1\right)$. Let $V_{S}$ be $|S| \times 1$ normalized noise power vector such that $i^{t h}$ entry of $V_{S}$ is $\beta_{i} N_{0} / g_{i i}$. Then, there exists a feasible power vector if and only if the greatest real eigenvalue of $D_{S} B_{S}$ is less than 1 and every element of the component-wise minimum power vector $\left(I-D_{S} B_{S}\right)^{-1} V_{S}$ is less than or equal to $P^{\max }$. The feasibility of the power vector $\left(I-D_{S} B_{S}\right)^{-1} V_{S}$ is further checked for the given EH time $\tau^{0}$ by ensuring whether its every element satisfies Eq. (3c).

The optimal time slot length, $\tau$, of Problem (3), is then found via bi-section search, where the feasibility of the mid-point of the search interval at each iteration is checked as described above. The initial lower and upper bounds of the interval are set as the maximum infeasible and minimum feasible values for $\tau$. To determine these values, we define the following three special cases:

$$
\begin{gathered}
\tau_{\text {min }}^{D}=\max _{i \in S}\left(D_{i} / W \log _{2}\left(1+P_{i}^{\max } g_{i i} / W N_{0}\right)\right), \\
\tau_{\min }^{E}=\min _{i \in S}\left(E_{i} \tau_{0} / P_{i}^{\max }\right), \\
\tau_{\max }^{E}=\min _{i \in S}\left(E_{i} \tau_{0} / \gamma\right) .
\end{gathered}
$$

Eq. (4) computes the maximum value of the required IT times, when only one user transmits with the maximum transmit power $P^{\max }$, while assuming that the users have enough energy for the described IT. Eq. (5) is the minimum of the IT times, where users transmit at $P^{\max }$ and is limited by the harvested energy amount. This is the shortest time duration that a user can transmit with the harvested energy. Eq. (6) is the minimum of the IT times, where users transmit at a low power level $\gamma$, which is a small and positive constant, and IT time is limited by the harvested energy amount. This is the longest time duration that a user can transmit with the harvested energy.

FIPCA-EH, given in Algorithm 1, is described as follows: The algorithm computes $\tau_{\min }^{E}, \tau_{\max }^{E}$, and $\tau_{\min }^{D}$ for a given $\tau_{0}$ (Line 1), and determines the lower and upper bounds for the bisection search (Lines 2-10). Then, bi-section search is performed by checking the feasibility of the middle of the interval at each iteration and updating the bounds accordingly (Lines 11-16).

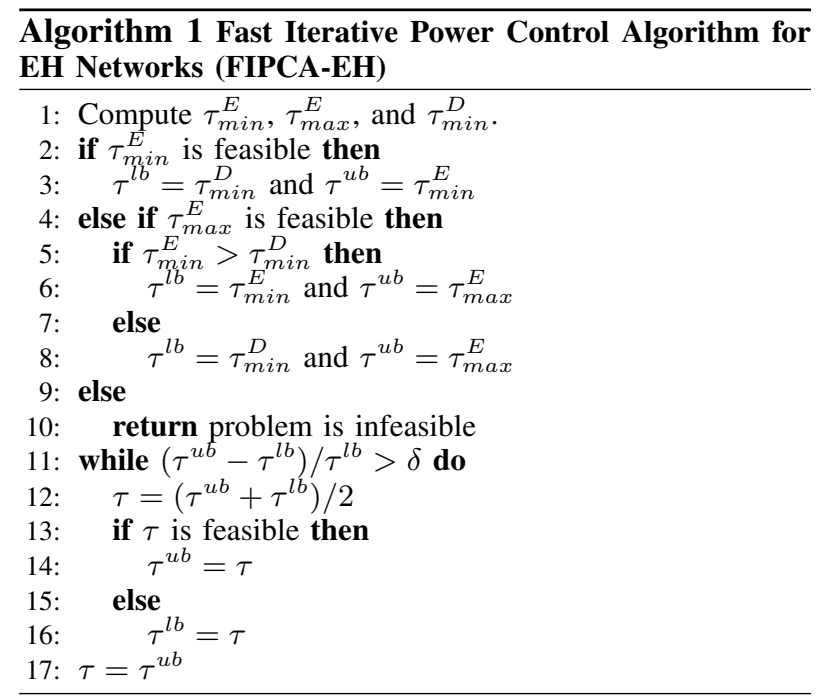

\section{Scheduling Problem}

Once the minimum time slot lengths of all possible simultaneous IT scenarios are calculated by the power control problem, the minimum length scheduling problem is formulated by assigning a variable to each of these scenarios, i.e., $\epsilon=\left\{\epsilon_{k}: 1 \leq k \leq|\epsilon|\right\}$ is the set of all feasible simultaneous IT scenarios of the link set $L=\{1,2, \ldots, L\}$.

The scheduling problem is an integer programming (IP) problem and it is given in Eq. (7) as follows:

$$
\begin{aligned}
\min & t^{T} c \\
\text { s.t. } & Q c \geq \mathbf{1}, \\
\text { var } & c^{k} \in\{0,1\}, \quad k \in[1,|\epsilon|] .
\end{aligned}
$$

where $t$ is an $|\epsilon| \times 1$ vector and $k$-th element of $t, t^{k}$ is the minimum time slot length corresponding to $\epsilon_{k}, Q$ is an $L \times|\epsilon|$ matrix and $Q_{l k}$ takes the value of 1 if link $l$ transmits its data in the $k$-th simultaneous IT scenario and of 0 otherwise. 1 in the right-hand-side of Eq. (7b) represents the all ones vector. $c$ is an $|\epsilon| \times 1$ vector and $k$-th element of $c, c^{k}$ is the binary variable which equals to 1 if $\epsilon_{k}$ exists in the optimal schedule and 0 otherwise. Eq. (7b) ensures that each link is scheduled at least once to transmit its data requirement.

Problem (7) is intractable, since determining the time slot length required for each possible transmission scenario requires an exponential effort. Even if the time slot lengths are known, the scheduling problem still has exponential number of integer variables.

We introduce a BP Based Heuristic Algorithm for EH Networks (BP-EH) to solve the intractable scheduling problem given in Eq. (7). BP-EH uses CGM to solve 
the LP relaxation of this IP problem at each node of the Branch and Bound tree.

BP-EH starts with the exponential IP formulation of Problem (7). The LP relaxation of the problem is solved using CGM. If the solution is fractional, one of the fractional variables is selected and set to either 1 or 0 to form two new IP problems. This is called branching.

After branching, the LP relaxation of two newly formed problems are solved by CGM iteratively. Branching on a node is stopped ( $i$ ) if the solution provided by CGM at that node is infeasible, (ii) if the solution is integral, in that case, the best integral solution is updated, and (iii) if the solution is fractional and it's worse than the best integral solution. Otherwise, branching is continued until there is no remaining node to branch on.

CGM is generally used for solving large-scale IP problems and LP relaxation of the IP problems at the nodes of the BP tree. CGM is solved by a heuristic algorithm, Reduced Cost Minimization Algorithm (RCMA) [14].

\section{Simulations and Numerical RESUlts}

The goal of the simulations is to compare the performance of the proposed algorithm, MAMUSA to POWMU [6], MAX-EH [6] and Transmission Completion Time-minimized Algorithm (TCT) [5] and evaluate the effect of the simultaneous transmission on the network performance. POWMU, MAX-EH and TCT schedule users' ITs for single AP multiple user EH networks where simultaneous transmission is not allowed. TCT is an optimal algorithm for the scheduling time minimization problem where there is no UL power limit on users, while POWMU and MAX-EH are optimal and heuristic algorithms for that problem with the additional UL power limit, respectively. In our simulations, we adopt these algorithms for multiple AP multiple user scenarios by allowing users to harvest energy from all the APs and scheduling their IT, based on HTT protocol. Further, we force TCT to obey the $P^{\max }$ limitation, i.e., for any user allocated to transmit at a power level greater than $P^{\max }$, its transmit power is adjusted to $P^{\max }$.

Simulations are performed over 100 independent random network topologies in MATLAB. A $8 \times 8 \mathrm{~m}^{2}$ area is divided into $C=4$ equally sized sub-regions and APs are centered in each sub-region. The users covered by each AP are uniformly distributed within the $4 \times 4$ $m^{2}$ sub-regions around their corresponding APs. Based on the observation of similar behaviour for networks with larger number of users, only one user assigned to each AP, i.e., $N=C$, for the sake of the simulation running time, unless otherwise stated. Simulation results are obtained for an indoor environment operating at a center frequency of $915 \mathrm{MHz}$ and a bandwidth of $1 \mathrm{MHz}$.

Both large and small scale statistics are considered to calculate the channel attenuation. The large scale fading is modeled by $P L(d)=P L\left(d_{0}\right)+10 \alpha \log \left(d / d_{0}\right)+Z$, where $d$ is the distance between the user and the AP.

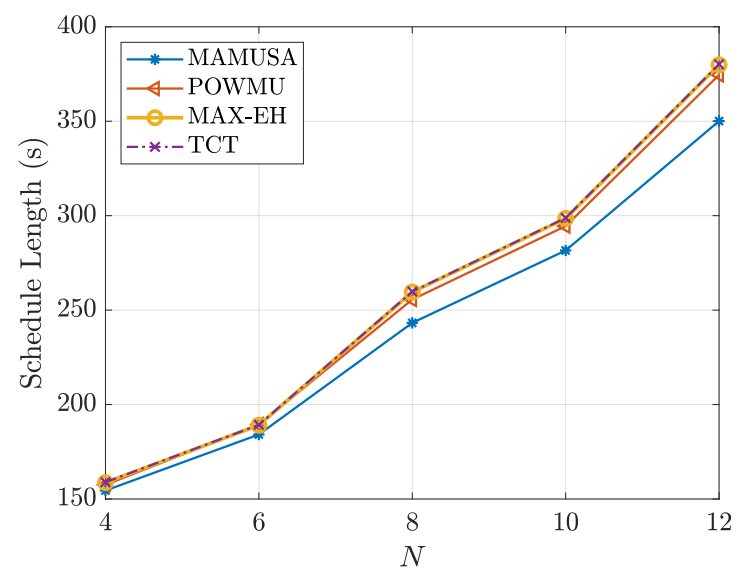

Fig. 1. Schedule length (s) vs the number of users.

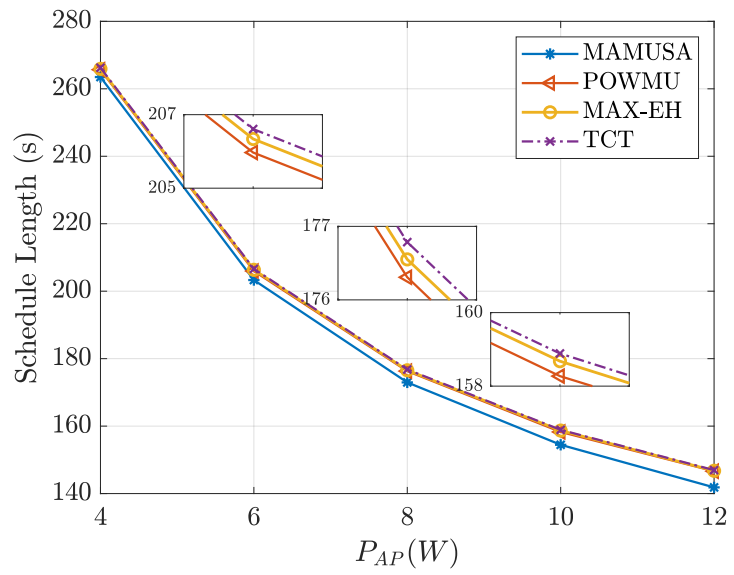

Fig. 2. Schedule length (s) vs transmit power (W) of APs.

$P L(d)$ and $P L\left(d_{0}\right)$ are the path loss in decibels $(\mathrm{dB})$ at distance $d$ and reference distance $d_{0}=1 \mathrm{~m}$, respectively. $\alpha$ denotes the path loss exponent [16], whereas $Z$ is a Gaussian random variable with zero mean and standard deviation $\sigma_{z}$. Rayleigh fading model is assumed for small scale fading with scale parameter equal to the mean power level determined by $P L(d)$ [17].

The simulation parameters are selected as follows: $N_{0}=10^{-7} \mathrm{~W} / \mathrm{Hz}, \sigma_{Z}=2 \mathrm{~dB}, P L\left(d_{0}\right)=31.7 \mathrm{~dB}$, $\alpha=2, \zeta=0.9, D_{i}=10$ bits $\forall i, P_{A P}=10 \mathrm{~W}$, and $P^{\max }=1 \mathrm{~mW}$.

Fig. 1 depicts the total schedule length for increasing number of users under APs. Obviously, the total schedule length increases with the increasing number of users. On the other hand, greater number of the users increases the gain from the simultaneous IT. MAMUSA provides $2.8 \%$ and $6.5 \%$ decrease in the total schedule length for $N=4$ and $N=12$, respectively.

Fig. 2 depicts the schedule length of a 4-AP 4-user network for varying $P_{A P}$ values. MAMUSA provides $3.5 \%$ 


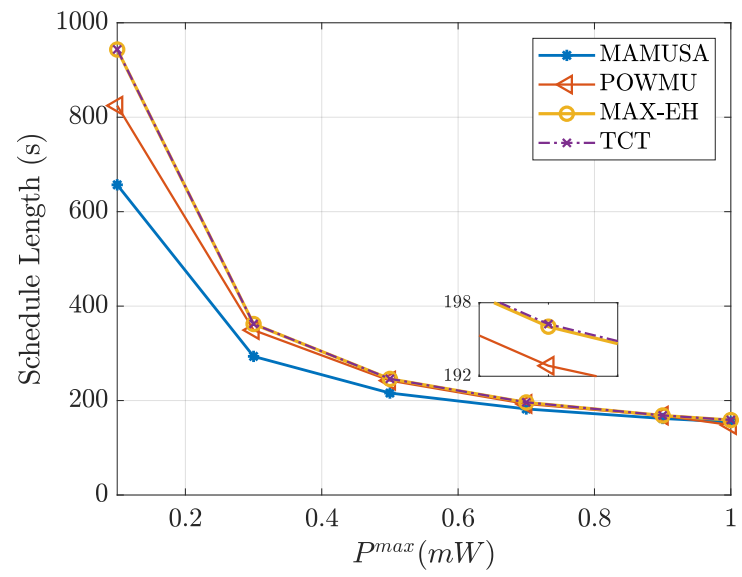

Fig. 3. Schedule length (s) vs the maximum UL transmit power (mW) of the users.

lower schedule length than MAX-EH for $P_{A P}=12 \mathrm{~W}$. As $P_{A P}$ decreases, the network needs more time for $\mathrm{EH}$ so the advantage of simultaneous IT also decreases.

Fig. 3 presents the schedule length of a 4-AP 4-user network for varying $P^{\max }$ values. MAMUSA outperforms benchmark approaches especially for lower $P^{\text {max }}$, e.g., $25.5 \%$ for $P^{\max }=0.1 \mathrm{~mW}$. This is as expected, since the lower the $P^{\max }$, the higher the IT times, so there is more room for improvement with simultaneous transmissions.

\section{CONCLUSION}

We analyzed the minimum length scheduling problem for a multi-cell HTT network with half-duplex, single antenna APs, and multiple, single antenna users, where the simultaneous UL IT is allowed. The EH and IT times, and UL transmit powers are allocated to optimize the scheduling length under minimum data and maximum transmit power limitations of the users. The problem is mixed integer, nonlinear, and non-convex.

We study the problem in two tiers and name the overall heuristic solution as MAMUSA. In the first tier, the $\mathrm{EH}$ time is assumed to be fixed and corresponding power allocations, IT times and schedule is found by further decomposing the problem into two problems, such as power control and scheduling. FIPCA-EH algorithm is proposed to solve the power control problem; whereas BP and CGM are used to solve the scheduling problem. In the second tier, the optimal EH time that minimizes the total schedule length is searched via simulated annealing.

MAMUSA is compared with three benchmark algorithms that provide solutions for single AP networks with no simultaneous transmission. Simulation results show that MAMUSA outperforms previously proposed algorithms up to $3.5 \%$ for larger AP power in DL EH,
$25.5 \%$ for tighter maximum power limit in UL IT, and $6.5 \%$ for greater number of users.

\section{REFERENCES}

[1] X. Lu, P. Wang, D. Niyato, D. I. Kim, and Z. Han, "Wireless networks with rf energy harvesting: A contemporary survey," IEEE Communications Surveys Tutorials, vol. 17, no. 2, pp. 757789, Secondquarter 2015

[2] F. Shan, J. Luo, W. Wu, and X. Shen, "Delay minimization for data transmission in wireless power transfer systems," IEEE Journal on Selected Areas in Communications, vol. 37, no. 2, pp. 298-312, Feb 2019.

[3] X. Kang, C. K. Ho, and S. Sun, "Full-duplex wireless-powered communication network with energy causality," IEEE Transactions on Wireless Communications, vol. 14, no. 10, pp. 55395551, Oct 2015.

[4] M. S. Iqbal, Y. Sadi, and S. Coleri Ergen, "Minimum length scheduling for discrete rate based full duplex wireless powered communication networks," in Ad-Hoc, Mobile, and Wireless Networks, M. R. Palattella, S. Scanzio, and S. Coleri Ergen, Eds. Cham: Springer International Publishing, 2019, pp. 343-354.

[5] K. Chi, Y. Zhu, Y. Li, L. Huang, and M. Xia, "Minimization of transmission completion time in wireless powered communication networks," IEEE Internet of Things Journal, vol. 4, no. 5, pp. 1671-1683, Oct 2017.

[6] E. D. Salik, A. Gurur Onalan, and S. C. Ergen, "Minimum length scheduling for power constrained harvest-then-transmit communication networks," in 2019 IEEE 30th Annual International Symposium on Personal, Indoor and Mobile Radio Communications (PIMRC), Sep. 2019, pp. 1-6.

[7] Z. Yu, K. Chi, K. Zheng, Y. Li, and Z. Cheng, "Transmit power allocation of energy transmitters for throughput maximisation in wireless powered communication networks," IET Communications, vol. 13, no. 9, pp. 1200-1206, 2019.

[8] C. Guo, B. Liao, and L. Huang, "Time allocation and load balancing in multi-cell wireless powered communication networks," IEEE Access, vol. 4, pp. 7795-7805, 2016.

[9] H. Kim, H. Lee, L. Duan, and I. Lee, "Sum-rate maximization methods for wirelessly powered communication networks in interference channels," IEEE Transactions on Wireless Coтmиnications, vol. 17, no. 10, pp. 6464-6474, Oct 2018.

[10] C. He, J. Liang, G. Qian, C. Guo, and D. Feng, "Optimal time allocation in multi-cell wireless powered communication networks," IEEE Access, vol. 7, pp. 26519-26 526, 2019.

[11] J. Liang, D. Feng, C. He, G. Qian, C. Guo, and N. Zhang, "Joint time and power allocation in multi-cell wireless powered communication networks," IEEE Access, vol. 7, pp. $43555-$ $43563,2019$.

[12] A. S. Sengupta, S. Satpathy, S. P. Mohanty, D. Baral, and B. K. Bhattacharyya, "Supercapacitors outperform conventional batteries [energy and security]," IEEE Consumer Electronics Magazine, vol. 7, no. 5, pp. 50-53, Sep. 2018.

[13] S. Boyd and L. Vandenberghe, Convex Optimization. USA: Cambridge University Press, 2004.

[14] Y. Sadi and S. C. Ergen, "Minimum length scheduling with packet traffic demands in wireless ad hoc networks," IEEE Transactions on Wireless Communications, vol. 13, no. 7, pp. 3738-3751, July 2014.

[15] R. Carr. Simulated annealing. MathWorld - A Wolfram Web Resource, created by Eric W. Weisstein. Accessed: 2020-02-13. [Online]. Available: http://http://mathworld.wolfram.com/SimulatedAnnealing.html

[16] S. Y. Seidel and T. S. Rappaport, "914 MHz path loss prediction models for indoor wireless communications in multifloored buildings," IEEE Transactions on Antennas and Propagation, vol. 40, no. 2, pp. 207-217, Feb 1992.

[17] R. Morsi, D. S. Michalopoulos, and R. Schober, "Multiuser scheduling schemes for simultaneous wireless information and power transfer over fading channels," IEEE Transactions on Wireless Communications, vol. 14, no. 4, pp. 1967-1982, April 2015. 